\title{
Preferred Orientation of Calcium Aluminosilicate Hydrate Induced by Confined Compression
}

\author{
Guoqing Geng ${ }^{1,2^{*}}$, Roman Nikolayevich Vasin ${ }^{3}$, Jiaqi Li $^{1}$, Mohammad Javad \\ Abdolhosseini Qomi ${ }^{4}$, Jinyuan Yan ${ }^{5}$, Hans-Rudolf Wenk ${ }^{6}$, Paulo J. M. Monteiro ${ }^{1}$ \\ ${ }^{1}$ Department of Civil and Environmental Engineering, University of California, \\ Berkeley, California 94720, United States \\ ${ }^{2}$ Laboratory for Waste Management, Paul Scherrer Institut, 5232 Villigen PSI, \\ Switzerland \\ ${ }^{3}$ Frank Laboratory of Neutron Physics, Joint Institute for Nuclear Research, Joliot-Curie \\ 6, 141980 Dubna, Moscow Region, Russia \\ ${ }^{4}$ The Henry Samueli School of Engineering, University of California, Irvine, California \\ 92697, United States \\ ${ }^{5}$ Advanced Light Source, Lawrence Berkeley National Laboratory, Berkeley, California \\ 94720, United States \\ ${ }^{6}$ Department of Earth and Planetary Science, University of California, Berkeley, \\ California 94720, United States \\ *Corresponding author. E-mail: guoqing.geng@ psi.ch.
}

\begin{abstract}
The existing macroscale models of the calcium (alumino)silicate hydrate (C-(A-)S-H), the main binder of concrete, assume that the nanocrystallites maintain random orientation under any loading conditions. However, using synchrotron-radiation-based XRD, we report the development of preferred orientation of nanocrystalline C-A-S-H, from random
\end{abstract}


at ambient pressure to strongly oriented under uniaxial compression with lateral confinement. The $c$-axes of the nanocrystals tend to align with the primary load. This preferred orientation is preserved after removing of external loading. The texture, quantified using a standard Gaussian fiber orientation distribution function (ODF), was used to calculate the averaged bulk elastic tensor of oriented C-(A-)S-H. It changes from isotropic (without texture) to transversely isotropic (with texture). Our results provide direct evidence of the reorientation of nanocrystalline $\mathrm{C}-(\mathrm{A}-) \mathrm{S}-\mathrm{H}$ as a mesoscale mechanism to the irreversible deformation of cement-based material. The implications of these results for modeling the mechanical property of $\mathrm{C}-(\mathrm{A}-) \mathrm{S}-\mathrm{H}$ at the macroscale are discussed.

Keywords: Calcium Aluminosilicate Hydrate, Texture Formation, High Pressure X-Ray Diffraction, Deviatoric Stress, Elastic Moduli

\section{Introduction}

Quantifying the nano- and micro-scale structure of cement-based materials is a key to understanding, designing and, most importantly, controlling their macroscale properties [1]. This endeavor has met great challenges when applied to calcium silicate hydrate $(\mathrm{C}-\mathrm{S}-\mathrm{H}$, $\mathrm{C}=\mathrm{CaO}, \mathrm{S}=\mathrm{SiO}_{2}, \mathrm{H}=\mathrm{H}_{2} \mathrm{O}$ in cement chemistry notation), which is the major binding phase in Porland cement (PC) concrete. This is largely due to its hierarchically porous structure, poorly crystalline nature and highly variable chemical composition [2]. For instance, the calcium-to-silica ratio $(\mathrm{Ca} / \mathrm{Si})$ of $\mathrm{C}-\mathrm{S}-\mathrm{H}$ ranges from $\sim 0.6$ to $\sim 2.0$ depending on the initial raw material composition $[3,4]$. C-S-H also uptakes significant amounts of Al 
to form $\mathrm{C}-\mathrm{A}-\mathrm{S}-\mathrm{H}\left(\mathrm{A}=\mathrm{Al}_{2} \mathrm{O}_{3}\right)$, as found in $\mathrm{PC}[5,6]$ and ancient Roman concrete [7-9] blended with aluminous pozzolanic materials.

Nonetheless, researchers have proposed structural models of $\mathrm{C}-(\mathrm{A}-) \mathrm{S}-\mathrm{H}$ that span length scales from nanometers to microns. For synthesized type I C-(A-)S-H, over the $\mathrm{Ca} / \mathrm{Si}$ range from 0.6 to $2.0, \mathrm{C}-(\mathrm{A}-) \mathrm{S}-\mathrm{H}$ remains a poorly crystalline layer structure highly analogous to tobermorite minerals, which is composed of $\mathrm{CaO}_{7}$ sheet being "sandwiched" by silicate tetrahedra chains $[10,11]$. This silicate chain, also termed dreierketten chain, is composed of periodically repeated pair silicate tetrahedra, connected by bridging silicate tetrahedra (Fig. 1). As the $\mathrm{Ca} / \mathrm{Si}$ ratio increases, the bridging silicate tetrahedra are increasingly omitted along with the enrichment of $\mathrm{Ca}$ in the interlayer (Fig. 1) [12-17]. C(A-)S-H usually exhibit a poorly-ordered layer stacking along $c$-axis [18-19]. Such stacking seems to be more disordered when $\mathrm{Al}$ is uptaken at low curing temperatures, whereas it is much more ordered at higher curing temperatures due to the Al-induced crosslinking, although the crystallite size is still limited to a few nanometers (Fig. 1) [18,20]. The C-(A-)S-H formed from PC systems has similar layer structure as type I C-(A-)S-H but the layer stacking is more disordered, such that it displays only one broad diffraction peak [21] at the location where type I C-(A-)S-H yields the strongest diffraction, i.e. of Laue indices $(020)$ and $(022)$ as refined by the $11 \AA$ tobermorite structure (monoclinic, $\left.a=6.735 \AA, b=7.385 \AA, c=22.487 \AA, \gamma=123.25^{\circ}\right)[10,11,20,21]$. Note that several tobermorite-based structural models can be used to refine the C-(A-)S-H lattice parameters and yield almost identical results [20]. Throughout the paper, C-(A-)S-H will be used to denote the tobermorite-like nanocrystalline $\mathrm{C}-\mathrm{S}$-H, either with or without Al-incorporation. 
C-A-S-H is specifically used to denote the Al-incorporated C-S-H, for example the sample being studied in this work.

In general, even the hotly debated nanoscale models of C-(A-)S-H in PC concrete [2,22-24] agree on the poorly-crystalline nature of $\mathrm{C}-(\mathrm{A}-) \mathrm{S}-\mathrm{H}$, whose smallest coherent domain size is estimated to be smaller than $5 \mathrm{~nm}$. This coherent domain size is consistent with pair distribution function studies [25] and nanoindentation results [26]. Historically, several names were used to denote such coherent domain in C-(A-)S-H, e.g. nano-globule [2], nano-grain [25], nano-platelet [27] and nanocrystal [13,14]. In the present work, the term 'nanocrystal' will be used throughout to denote the coherent region exhibiting a layered poorly-crystalline structure similar to tobermorite and often with the smallest dimension along the direction perpendicular to the layer. Atomistic modeling has been intensively applied in the past decade to correlate the chemical composition with the mechanical properties of C-(A-)S-H [28-33]. It is only recently that such correlations were directly verified by experimental data at molecular scale $[18,20]$. The bulk modulus of C(A-)S-H is greatly controlled by the compliance along its $c$-axis. The interlayer densification and the $\mathrm{Al}$-induced crosslinking are the dominant driving force for $\mathrm{C}-(\mathrm{A}-) \mathrm{S}$ $\mathrm{H}$ stiffening, whereas the bridging silicate omission is irrelevant to the overall stiffness $[18,20]$. 
Tobermorite

bridging site
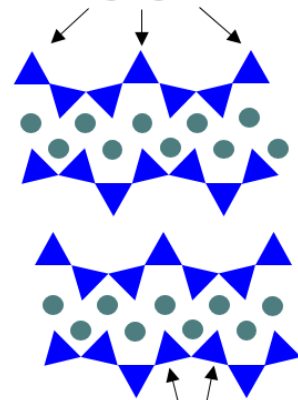

pair site
$\mathrm{Ca}$ uptaking

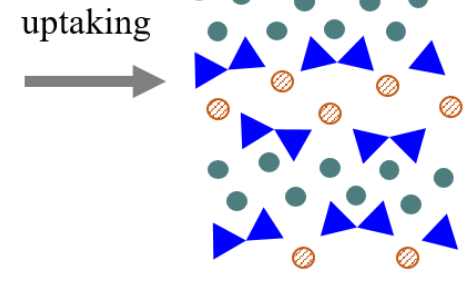

.
C-S-H
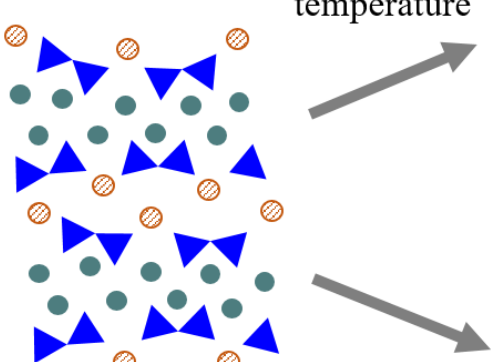

Al uptaking at high curing temperature
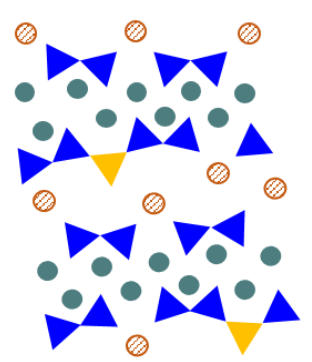

\section{C-A-S-H}

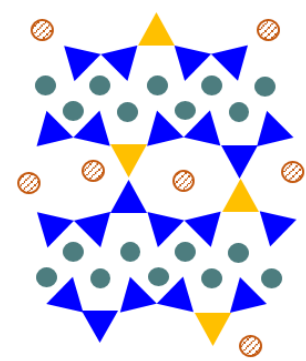

Fig. 1. Schematic layer-structural model of C-(A-)S-H and its evolution after uptaking $\mathrm{Ca}$ and $\mathrm{Al}$. The structural disorder in C-S-H and low-temperature C-A-S-H is illustrated. The $\mathrm{Al}$-induced crosslinking in high-temperature C-A-S-H improves the structural order. The water content is a function of the drying conditions and is not shown for viewing convenience.

The orientation distribution of grains in polycrystalline materials, often referred to as "texture", markedly determines the anisotropy of properties at the macroscale [34]. To our knowledge, the existing cement models, either based on hydration chemistry [35-37], or colloidal packing behavior [38], assume a random orientation of the C-(A-)S-H nanocrystals, which leads to isotropy of the matrix at the macroscale. It is therefore of interest to quantify the orientation of the nanocrystalline $\mathrm{C}-(\mathrm{A}-) \mathrm{S}-\mathrm{H}$ and to determine its influence on the anisotropy of C-(A-)S-H properties at the macroscale.

One of the most efficient ways to quantify grain orientation distributions is by studying the variation of intensity along the azimuthal angle of Debye rings obtained from synchrotron X-ray diffraction experiments. Once the texture is measured, homogenization 
models can be used to estimate the anisotropic properties at the macroscale [39-42]. The primary goal of the present work is to investigate whether nanocrystalline C-(A-)S-H develop preferred orientation under deviatoric stresses, to measure its evolution with increasing stresses, and to model how the texture influences the macroscopic elastic properties of C-(A-)S-H aggregate. Our previous work used synchrotron X-ray diffraction at high pressure (HP-XRD) to measure the mechanical properties of the $\mathrm{C}-(\mathrm{A}-) \mathrm{S}-\mathrm{H}$ nanocrystals in a diamond anvil cell (DAC) under hydrostatic pressure [18,20]. However, the development of texture in the polycrystalline material often requires the presence of deviatoric stress [43]. In the present work a hydrostatic pressure medium was not used in the DAC. The study of texture in diffraction experiments requires a sufficient crystallinity, therefore a C-A-S-H sample synthesized at $80{ }^{\circ} \mathrm{C}$ was selected because it was found to have the most ordered crystal structure among reported $\mathrm{C}-(\mathrm{A}-) \mathrm{S}-\mathrm{H}$ samples. Using the texture and a single crystal elastic constants as input, anisotropic elastic properties of C(A-)S-H at the macroscale were calculated. Note that the term "texture" in cement research community often refers to "microstructure" and is irrelevant to crystal preferred orientation [38-44]. In this work, "texture" specifically refers to the orientation distribution of nanocrystalline C-(A-)S-H.

\section{Materials and methods}

\subsection{Materials}

Details of C-(A-)S-H synthesis are reported elsewhere [20,45]. Stoichiometric amounts of $\mathrm{SiO}_{2}$ (Aerosil 200, Evonik), $\mathrm{CaO}$ (obtained by burning $\mathrm{CaCO}_{3}$ (Merck Millipore) at $1000{ }^{\circ} \mathrm{C}$ for 12 hours) and $\mathrm{CaAl}_{2} \mathrm{O}_{4}$ were mixed with water (water/solid ratio of 45 ), such 
that the initial $\mathrm{Ca} / \mathrm{Si}$ and $\mathrm{Al} / \mathrm{Si}$ molar ratios were 1.0 and 0.1 , respectively. The suspensions were then stored in a Teflon bottle at $80^{\circ} \mathrm{C}$ for 8 weeks. The reaction product was vacuum filtered and freeze-dried for 7 days. All the above processes were carried out in a $\mathrm{N}_{2}$-filled glove box to avoid carbonation. Following the notation in a previous publication, ${ }^{20}$ the sample is named hereafter Al10.

\subsection{HP-XRD in radial diffraction geometry}

The HP-XRD experiment was conducted at beamline 12.2.2 of the Advanced Light Source (ALS) at the Lawrence Berkeley National Laboratory (LBNL) [46], using a BX90 DAC [47]. As shown in Fig. 2a, to probe as many crystal orientations relative to the compression direction as possible, a radial geometry DAC was chosen where the incident beam $(Y$ direction) is perpendicular to the DAC axis (Z-direction), which is also the principal loading direction. The gasket was made by laser-drilling a $100-\mu \mathrm{m}$-diameter hole on a 80 $\mu \mathrm{m}$-thick boron epoxy resin piece, which was then inserted into a Kapton film [48]. The sample was loosely packed into the gasket hole (Fig. 2b). Since no pressure medium was used, the stress in the sample was initially zero, which began to build up as soon as the inter-particle porosity was essentially eliminated by the increasing compressive load (applied along Z-direction) [43]. Throughout the paper, the macroscale sample coordinate system will be denoted by $X, Y$ and $Z$, and the microscopic scale monoclinic single crystal coordinate system will be denoted by the unit cell directions $a, b$ and $c$, where $c$ is perpendicular to the $a b$-plane.

The load was applied by slowly tightening the load screws of the DAC. A thin piece of platinum $(\mathrm{Pt})$ plate was placed between one diamond anvil culet and the sample powder. At every pressure step, the diffraction peaks of Pt were analyzed to calibrate the pressure 
and deviatoric stresses in the sample chamber (Fig. 2b) [49]. We assume that the same stresses, i.e. not only the axial stress but also the other components, are shared between the C-A-S-H solid and the Pt grains. This Reuss approximation (equal stress in every grain) is often considered in geophysics research using high-pressure DAC in radial geometry [50]. High deviatoric stresses can develop in a DAC so it is relevant to check if the Pt grains have not yielded. Dorfman et al. reported that the yielding of platinum strongly depends on the loading conditions [51], the grain size and it can happen at differential stress (S/3 following our definition) values below $1 \mathrm{GPa}$. In the present experiment, the values of the deviatoric stress are smaller than $\sim 300 \mathrm{MPa}$, and there is no evidence of intensity redistribution along Pt Debye rings, which would have indicated the onset of the plastic deformation. Therefore our estimations of deviatoric stresses in C-(A-)S-H under pressure should not be significantly biased.

Considering the symmetry of the experimental setup, the macroscopic elastic stress tensor has the following non-zero components in the sample coordinate system (Fig. 2a): $\sigma_{x x}=\sigma_{y y}=P-S, \sigma_{z z}=P+2 S$, where $P$ is pure hydrostatic component (pressure), and $S$ is the deviatoric component of the stress $[50,52]$. Note that the stress state in the sample chamber could also be represented as a combination of a hydrostatic compression of $P-S$ and a pure uniaxial compression of $3 S$ along the $Z$-axis. Here, positive values are used for compression.

A MAR345 area detector was used to record diffraction images of the sample with a $300 \mathrm{~s}$ exposure for each diffraction measurement. Using a diffraction image of a $\mathrm{CeO}_{2}$ standard, the wavelength of the synchrotron X-ray was refined to $0.49755 \AA$, and the sample to detector distance to $331.6 \mathrm{~mm}$. We first measured the diffraction of Al10 sample 
under ambient condition (prior to loading) in axial geometry (where there is minimal scattering from the gasket material), i.e., rotating the cell to the orientation with incident beam along Z-direction. Following that, we switched to axial geometry (Fig. 2a) and recorded four diffraction images on the loading path from $P=1.3$ to $11.9 \mathrm{GPa}$, and four images on the unloading path down to $P=1.5 \mathrm{GPa}$. Note that at the end of the unloading path, when the external compressive load was completely removed, the sample still was under residual compression, i.e., $P=1.5$ GPa. The refined $P$ and $S$ values are shown in Fig. 2c.

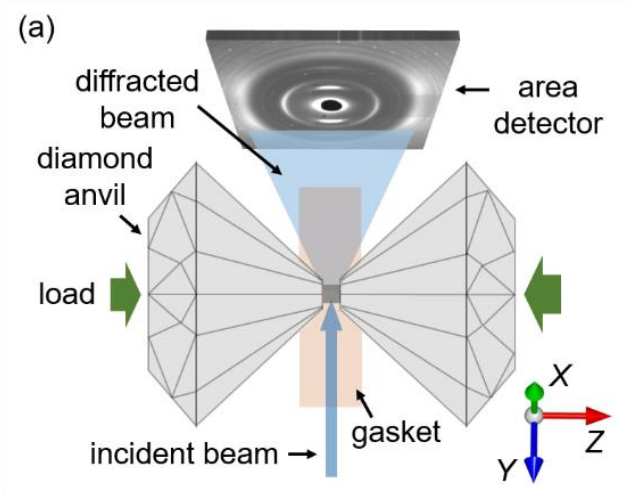

(b)

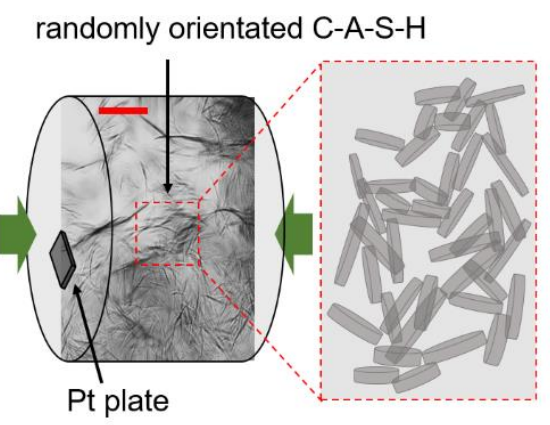

(c)

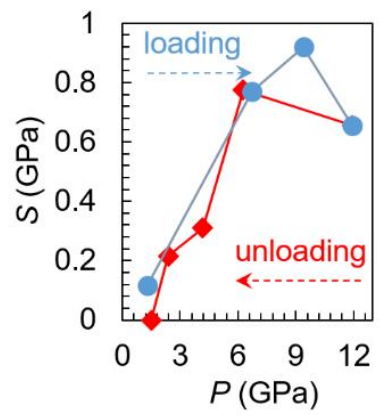

Fig. 2. Scheme of (a) the radial diffraction geometry using a DAC, (b) magnified picture of the randomly oriented loosely-packed nanocrystalline C-A-S-H in the sample chamber. Directions $Z$ and $Y$ are aligned with the DAC axis and incident beam path, respectively. The micro-morphology in (b) is adopted from a transmission image of Al10 at ambient condition (see previous research) [20]; scale bar (red) is $1 \mu \mathrm{m}$. The direction of the compressive load is indicated by the green arrows in both (a) and (b). The sample also experienced lateral confinement from the gasket. (c) The refined hydrostatic $(P)$ and deviatoric $(S)$ stress components in the sample along the loading path are denoted by the blue dots, and along the unloading path - by the red diamonds.

\subsection{Texture analysis and homogenization scheme}


Multiple tobermorite crystal structures have been proven suitable in refining the XRD results of Type I C-(A-)S-H [20]. The present study used the monoclinic $11 \AA$ tobermorite structure ( $\left.a=6.735 \AA, b=7.385 \AA, c=22.487 \AA, \gamma=123.25^{\circ}\right)$ [10] for the Rietveld refinement [53] performed using the MAUD software package [54]. As shown later on, the (002) peak is the only resolved peak from the sample at most elevated pressure values, therefore the unit cell length $c$ was the only refined lattice parameter. The unique peak broadening of type I C-(A-)S-H was accounted for by refining the anisotropic crystalline domain sizes, as enabled in MAUD [54]. For texture refinement using $M A U D$, the raw 2D diffraction image was segmented into 36 sectors, each spanning $10^{\circ}$ in the azimuthal angle $\eta$ (Fig. 3 a and $b)$. The conventional diffraction pattern for each sector was obtained by integration over $\eta$ and was then used as input for texture analysis.

Multiple texture models are imbedded in MAUD for texture quantification via Rietveld refinement, including representation of ODF by standard functions [55] and discrete WIMV and E-WIMV algorithms [56]; they have been used to refine the texture of ettringite in a hardened concrete matrix [57]. This study used a standard fiber function to describe the ODF developed in the nanocrystalline C-A-S-H. Preliminary data analysis revealed that Lorentzian content of the fiber and the amount of random texture component consistently converged to negligible values close to zero, thus they were omitted in final refinements and only the pure Gaussian fiber component was used to describe the ODF of C-A-S-H. The orientation of the fiber was constrained by the observed alignment of (001) crystal planes of C-A-S-H subnormal to the compression direction. To account for the possible misalignment of the DAC, the tilt of the fiber axis with respect to the sample coordinate system axis- $Z$ was also refined; however, in most cases it converged to rather 
small values, as will be discussed further. The width of the Gaussian fiber function was observed to vary with pressure and was refined. The E-WIMV texture model was used to refine the preferred orientation of Pt grains [56].

To determine the components of the elastic stress tensor, we used the Pt diffraction peaks. Following the accepted methodology [52], we assumed that hydrostatic part of the stress (i.e., pressure) is reflected in the unit cell volume change and is determined from unit cell parameter of Pt using the equation of state [49]. Then, the pressure-dependent stiffness tensor of Pt was used to refine deviatoric stress $S$. The so-called "BulkPathGEO" method [58], which is implemented in MAUD as a part of "Moment pole stress" model was used to recalculate deviatoric stress tensor components from diffraction peak shifts [52]. 
(a)

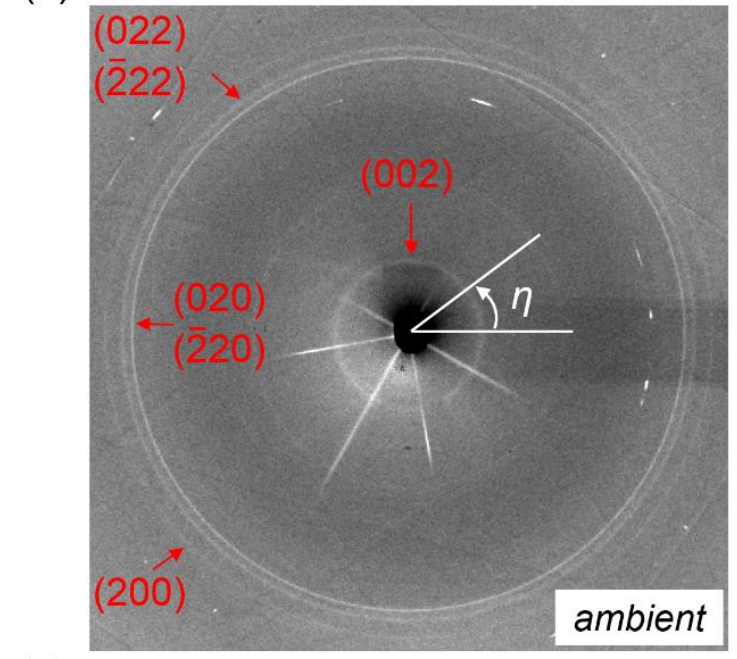

(c)

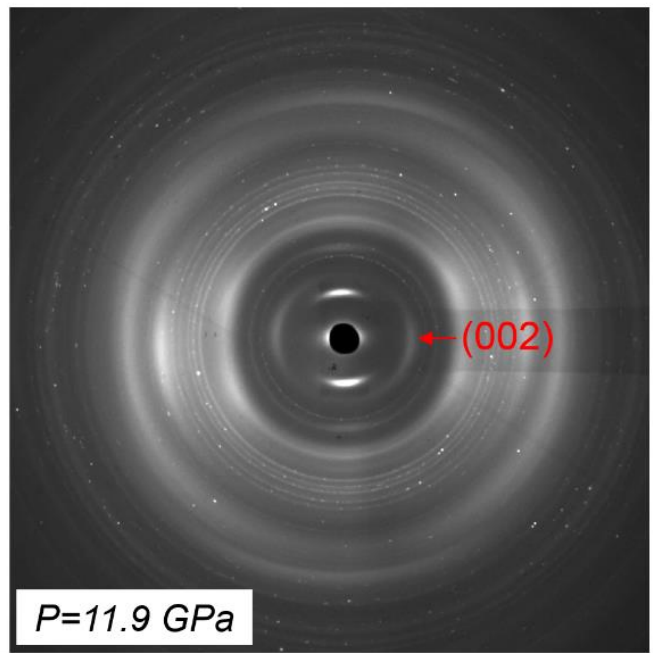

(b)

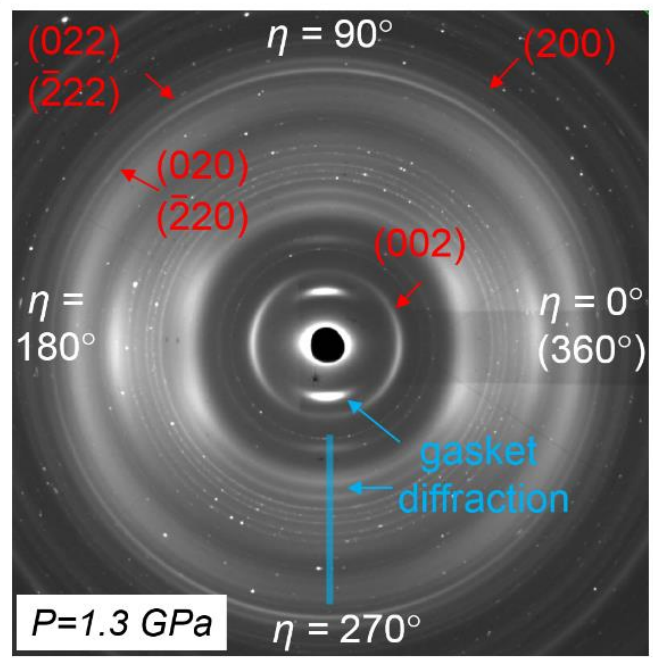

(d)

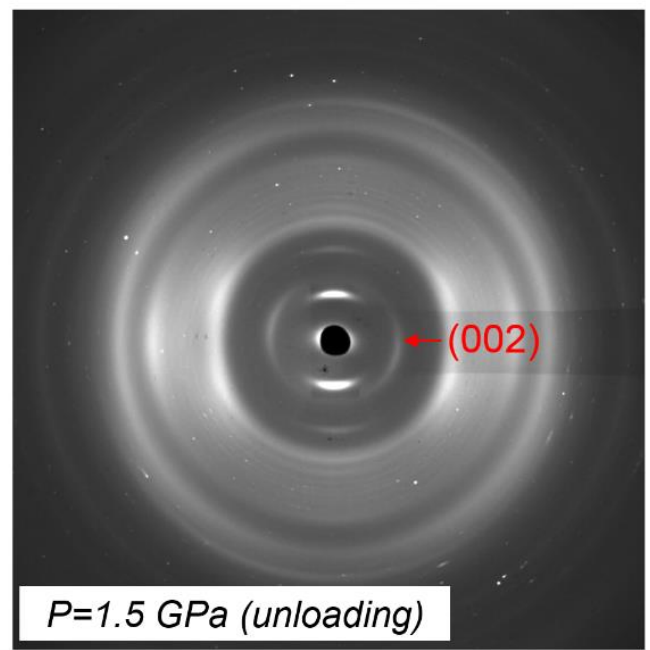

Fig. 3. Raw 2D diffraction patterns of the studied sample under (a) ambient condition, (b) $P=1.3 \mathrm{GPa}$, (c) $P=11.9 \mathrm{GPa}$ and (d) $P$ unloaded to $1.5 \mathrm{GPa}$. The C-A-S-H diffraction rings are marked with red arrows. The diffraction of gasket is absent in axial geometry (a), but present in the radial geometry (b-d), as marked by blue arrows and band. Some azimuthal angles $(\eta)$ are illustrated in (a) and (b) for viewing convenience. The primary load direction on the sample is close to the horizontal direction in the diffraction images.

Multiple methods are available to calculate bulk elastic properties of a textured polycrystalline material $[39,40]$. Herein, the modified self-consistent averaging scheme GEO-MIX-SELF (GMS) [41] was used to average the physical properties of the C-A-S-H 
at the macroscale. Compared to conventional self-consistent algorithms [59], GMS uses elements of the geometric mean approach to converge to a unique solution for bulk elastic properties [58], exactly obeying the inversion relation, i.e., stiffness is inverse compliance. Pores and cracks can be easily introduced into GMS scheme as voids approximated by ellipsoids to account for the porosity in C-A-S-H aggregate. It should be noted, that GMS models a polycrystalline aggregate of infinite (or at least very large) number of grains and consequently does not take grain sizes into account, only their shape.

In addition to texture and microstructure information, the single crystal stiffness tensor of C-A-S-H is necessary to compute bulk elastic properties. For Al10 material, it was obtained using the Lattice Harmonic Approximation (LHA) calculation with the GULP package [60]. To build molecular model of Al10, we began with a $2 \times 2 \times 1$ supercell of a crosslinked $11 \AA$ tobermorite model, whose bridging silicon atoms were partially replaced with aluminum atoms, to match the bulk $\mathrm{Al} / \mathrm{Si}$ ratio. The interlayer $\mathrm{Ca}$ was adjusted to match the bulk $\mathrm{Ca} / \mathrm{Si}$ ratio. No bridging site vacancies were included, because the silicate chain of Al10 was highly continuous as verified by NMR data [45]. The CSHFF force field was used here $[18,28,61]$, and the LJ-pair coefficients for Al were set identical to that of $\mathrm{Si}$, as is also adopted in the ClayFF force field [62]. Various charge balancing techniques were employed to distribute the charge difference around aluminum sites to ensure that the calculated data is not sensitive to charge neutralization scheme.

Using the calculated single-crystal elastic tensor of C-A-S-H, the effect of texture on the bulk elastic properties was analyzed. Finally, for comparison, the macroscale elastic tensor of a $\mathrm{C}-\mathrm{S}-\mathrm{H}$ matrix $(\mathrm{Ca} / \mathrm{Si}=1.0, \mathrm{Al} / \mathrm{Si}=0)$ was computed using the same ODFs as measured for C-A-S-H. As shown in our previous study, this C-S-H structure is 
considerably less stiff along the $c$-axis compared with its $a b$-plane, thus exhibiting strong elastic anisotropy [18]. This comparison highlights the influence of single-crystal elastic anisotropy on the macroscale properties of aggregated C-(A-)S-H with texture.

\section{Results}

\subsection{Texture formation and quantification}

At ambient condition, several diffraction rings on 2D images are from the sample (Fig. 3a) and corresponding Laue indices are assigned according to the $11 \AA$ tobermorite structure [20]. The absence of the abundant diffraction peaks of tobermorite is due to the nanocrystallinity of C-(A-)S-H. In the current synthesis condition, Al10 is more ordered along the $c$-axis than the $\mathrm{Al}$-free $\mathrm{C}-\mathrm{S}-\mathrm{H}$ samples and consequently the (002) diffraction ring is clearly resolved. The $(022)+(\overline{2} 22)$ diffractions are isolated from the $(020)+(\overline{2} 20)$ diffractions, whereas they merge into one broad peak for most reported C-S-H and lowtemperature C-A-S-H structures [13-15,18]. This is attributed to the Al-induced crosslinking at $80^{\circ} \mathrm{C}$ curing condition, which prevents the $a b$-plane gliding that would result in the peak broadening. Before applying load on the sample, the random orientation of the nanocrystalline regions is easily identified, as the intensity on the diffraction rings does not change with azimuthal angle (Fig. 3a).

Upon compression, the cylindrical sample chamber underwent inelastic shrinkage, accompanied by the close compaction of the aggregated Al10 within. Upon close contact of sample particles, $P$ began to build up. The first non-ambient $P$ that was picked up is calibrated to $1.3 \mathrm{GPa}$ (Fig. 3b), when a strong texture is readily observed from the sample diffraction. The basal diffraction (002) peak has strongest intensity close to the 
compression direction. On the contrary, the strongest intensity of the diffractions related to $a b$-plane, i.e. $(020)+(\overline{2} 20),(022)+(\overline{2} 22)$ and $(200)$, are perpendicular to the compression direction. As $P$ increases up to $11.9 \mathrm{GPa}$ and then decreases to $P=1.5 \mathrm{GPa}$, the (001) plane retains the same pattern of preferred orientation (diffraction images are available in the supporting information (S.I.)). Apart from the (002) peak, other peaks that were observed at ambient condition could not be resolved from the gasket scattering when $P$ is higher than 1.3 GPa, even after the decompression.

To quantify the preferred orientation of the (001), a standard Gaussian fiber model is used here. The term "fiber" can be misleading for a cement chemist. It does not refer to the morphology of single crystal or grain, but rather imply that the crystal orientation has a certain preferred axis ( $c$-axis in this study). Two parameters of the fiber model were refined, i.e., full-width-at-half-maximum (FWHM) and the angle defining the tilt of the Gaussian function from the $Y$-axis (ThetaY). As it will be shown later, ThetaY is often refined to a small non-zero value, which is due to the slight tilt of the DAC on the sample stage so that the compressive loading direction is not exactly along $Z$-direction. Note that the interpretation of the texture is merely based on the tobermorite-like mineralogical nature of nanocrystalline C-(A-)S-H, with no need to differentiate between the hotly debated colloidal model [2] or layer model [24].

As shown in Fig. $4 \mathrm{a}(P=1.3 \mathrm{GPa})$, the diffraction pattern of the sample (including Pt) is readily obtained by subtracting the scattering of the gasket (measured on the gasket location near the sample chamber) from the pattern of the sample loaded in gasket. This subtraction creates few small artifacts (highlighted by red dashed rectangles in Fig. 4a) in the background, which do not overlap with diffraction peaks of the sample. Consequently, 
to obtain the diffraction from the sample only, such a subtraction is applied to the whole 2D diffraction image, as shown in Fig. 4b. The resulting 2D image was then segmented into 36 diffraction patterns as described earlier. Of these 36 , only one half contains nonredundant information (due to the experiment symmetry, [52]). Thus, only patterns with $\eta$ values from $90^{\circ}$ to $270^{\circ}$ (Fig. 4c) were simultaneously analyzed in MAUD to extract the preferred orientations. In Fig. $4 b$, the illuminated area surrounding the black spot in the image center, as indicated by the yellow arrow, is due to the scattering of the beamstop whose intensity varies with $\eta$. During refinement, this beamstop scattering was represented by adding a Gaussian peak into the background, whose position and intensity were refined to match the experiment. 

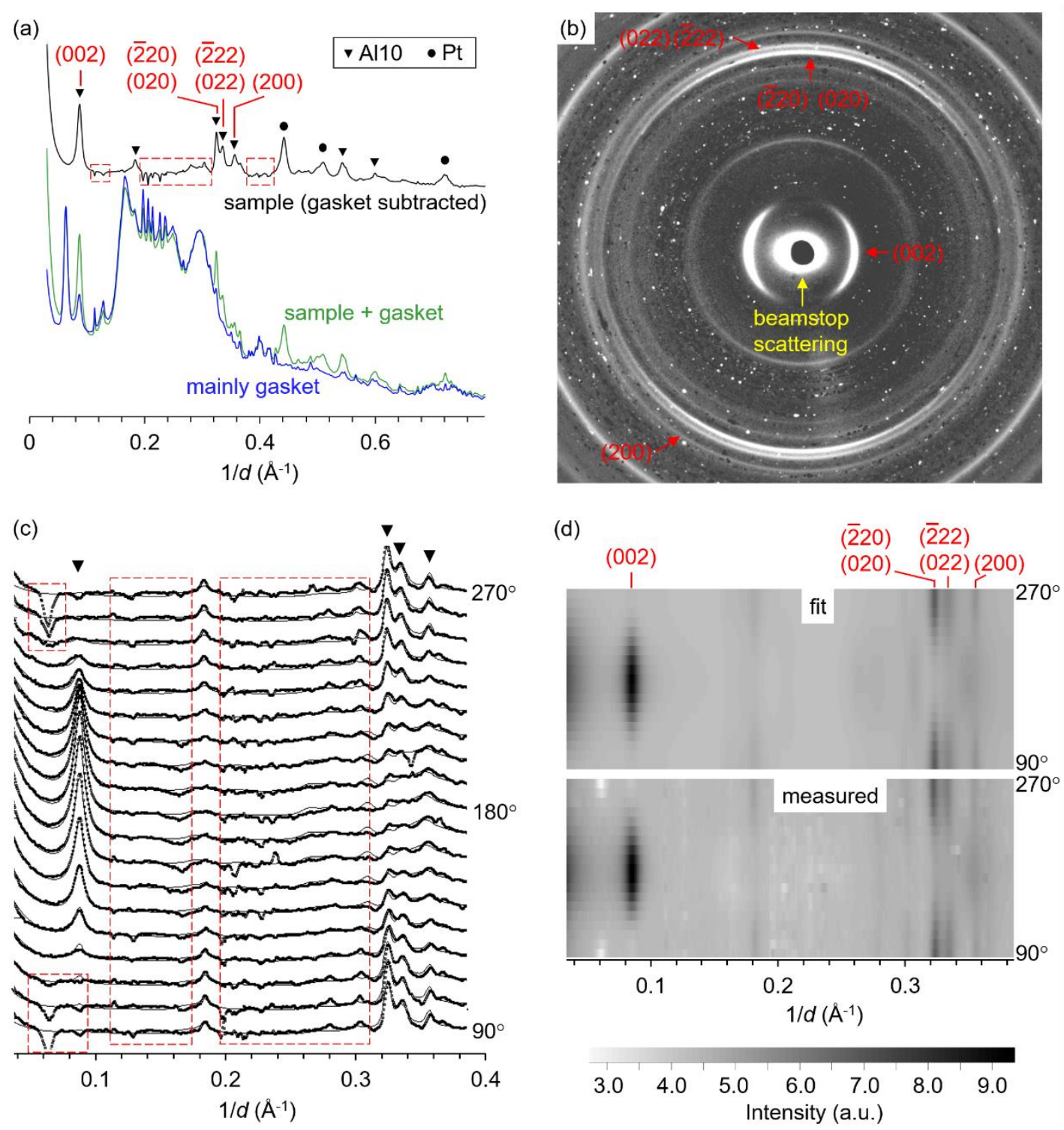

Fig. 4. Quantification of the texture at $P=1.3 \mathrm{GPa}$ using a $c$-fiber model. (a) integrated diffraction patterns of the gasket (with and without the sample), and of the sample only (via subtracting the gasket diffraction). (b) 2D diffraction image after subtracting the gasket diffraction from the sample image from Fig. 3b. Diffraction peaks of A110 and the scattering from beamstop are shown. (c) Measured diffraction patterns at different azimuthal angles (dots) and the fit results (thin solid lines). Red dashed boxes in (a) and (c) indicate the artifacts in the background that are generated by subtraction of the gasket signal. (d) Stacks of measured (bottom) and fitted intensities (top) as a function of azimuthal angle. 
Results of Rietveld refinement of C-A-S-H diffraction data at $P=1.3 \mathrm{GPa}$ are shown in Fig. $4 \mathrm{c}$ and d. A very good agreement of experimental and refined diffraction patterns is observed (excluding the artifacts in the background from the gasket signal subtraction), confirming the validity of the selected C-A-S-H structure model, as well as of the $c$-fiber model of texture. ThetaY is refined to $5.2^{\circ}$ and FWHM to $65.2^{\circ}$. The non-zero value of ThetaY indicates that the loading direction is slightly tilted relative to the $Z$-direction. The refined results completely describe the ODF the of A110 nanocrystals at $P=1.3 \mathrm{GPa}$. Based on this ODF, the preferred orientations of various lattice directions can then be determined (Fig. 5a).

(a)
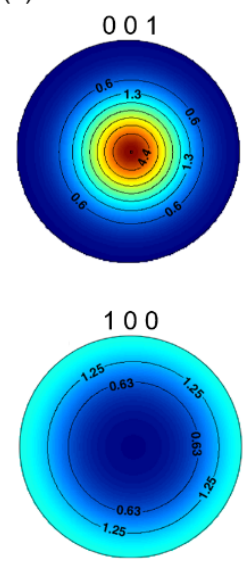

0.01 .02 .03 .04 .05 .0 (b)

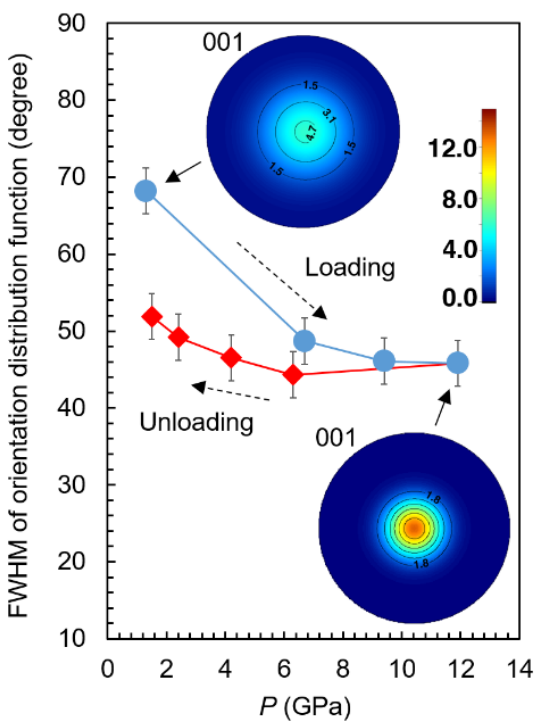

(c)

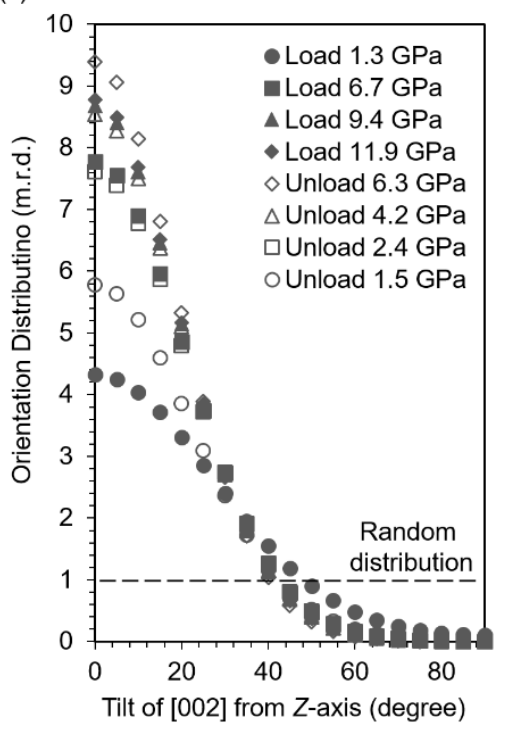

Fig. 5. (a) (Equal area projection) pole figures of C-A-S-H at $P=1.3 \mathrm{GPa}$ (loading path). The linear scale of orientation probability density is in m.r.d. (b) The refined FWHM of the $c$-fiber model as a function of $P$ on both loading (blue round symbols) and unloading path (red diamond symbols). The loading and unloading paths are indicated by dashed arrows. (c) Profiles of pole density on (001) pole figure, as a function of the angle between its normal and the $Z$-axis. The dashed line corresponds to the random distribution. 
Since the $c$-fiber model was confirmed to correctly fit the experimental data, only the part of diffraction patterns containing (002) peak of C-A-S-H was analyzed in MAUD for all other pressure points on the loading and unloading paths. The refined crystallographic and texture information is listed in Table 1 and illustrated in Fig. 5b. The FWHM of the orientation distribution monotonically decreases during the loading process, from $65.2^{\circ}$ at $P=1.3 \mathrm{GPa}$ to $45.8^{\circ}$ at $P=11.9 \mathrm{GPa}$. When the pressure is slowly released, there is a delay in the bounce-back of FWHM (red diamond symbols). When the load is completely removed, we observed a residual texture with FWHM of $51.9^{\circ}$. The orientation distribution of (001) is visualized as (001) pole figures for the completely unloaded state and the most loaded state (insets in Fig. 5b). The strongest orientation distribution of (001) is observed at $P=11.9 \mathrm{GPa}$.

For axially symmetric textures, the often used representation is a profile of pole densities from macroscopic fiber axis (in our case it is tilted by ThetaY to Z-axis) to edge with units of multiples of random distribution (m.r.d.). In Fig. 5c, these profiles are plotted for (001) pole figure as a function of the angle between [001] and the Z-axis. Interestingly, a residual deformation of $\mathrm{Pt}$ that corresponds to $P=1.5 \mathrm{GPa}$ is observed when the sample is completely unloaded. This indicates a residual strain in C-A-S-H matrix that prevented the imbedded Pt to become strain-free. Indeed, it is observed that the basal (002) spacing of the completely unloaded A110 to be $11.25 \AA$, compared with $11.4 \AA$ at ambient condition [20].

Table 1 Refined crystallographic and texture information of C-A-S-H at different pressures $P$ : unit cell parameter $c$, breadth FWHM and tilt ThetaY of Gaussian fiber texture, and maximum value Max on (001) pole figure.

\begin{tabular}{lllll}
\hline$P(\mathrm{GPa})$ & $c(\AA)$ & FWHM & ThetaY & Max (m.r.d.) \\
\hline
\end{tabular}




\begin{tabular}{cccccc}
\hline \multirow{5}{*}{ Loading } & 1.3 & $22.8 \pm 0.1$ & $65.2^{\circ} \pm 3^{\circ}$ & $5.2^{\circ}$ & 4.4 \\
& 6.7 & $21.5 \pm 0.1$ & $48.7^{\circ} \pm 3^{\circ}$ & $1.33^{\circ}$ & 7.8 \\
& 9.4 & $21.0 \pm 0.1$ & $46.1^{\circ} \pm 3^{\circ}$ & $0.4^{\circ}$ & 8.7 \\
& 11.9 & $20.7 \pm 0.1$ & $45.8^{\circ} \pm 3^{\circ}$ & $0.8^{\circ}$ & 8.8 \\
\hline \multirow{4}{*}{ Unloading } & 6.3 & $21.4 \pm 0.1$ & $44.3^{\circ} \pm 3^{\circ}$ & $1^{\circ}$ & 9.5 \\
& 4.2 & $21.7 \pm 0.1$ & $46.5^{\circ} \pm 3^{\circ}$ & $-0.3^{\circ}$ & 8.5 \\
& 2.4 & $22.0 \pm 0.1$ & $49.2^{\circ} \pm 3^{\circ}$ & $1.1^{\circ}$ & 7.6 \\
\hline
\end{tabular}

This study focused next on the nanocrystals whose $c$-axes are along the macroscale $Z$-axis, and investigated the integrated diffractogram from $\eta=175^{\circ}$ to $185^{\circ}$. The Biot strain $\left(\varepsilon=1-l / l_{0}\right)$ of the $c$-axis is plotted as a function of the hydrostatic component $P$, and compared with the reported $c$-axis strain under hydrostatic loading only [20] (Fig. 6a). The $c$-axis of these nanocrystalline $\mathrm{C}$-A-S-H develops nearly twice the amount of strain, compared with the C-A-S-H under merely hydrostatic condition. Notice that the stress state in the sample chamber may be represented as a superposition of a hydrostatic compression of $P-S$ and a uniaxial compression of $3 S$ along the $Z$-axis. For each data point in this study, we extrapolated the strain due to a hydrostatic compression of $P-S$ assuming an incompressibility of $1 /(240 \mathrm{GPa}$ ) (Fig. 6a), which is then subtracted from the overall strain to yield a strain difference $\varepsilon_{c}$. According to the superposition principle, $\varepsilon_{c}$ is merely generated by the uniaxial compression of $3 S$, as plotted in Fig. 6b. The loading and unloading curves of $\varepsilon_{c}$ seem to follow two lines with similar slopes, but there is evidence of a slight drift. This drift may indicate an atomic positional relaxation in the interlayer region that results in a permanent deformation of $\sim 1.5 \%$ along $c_{-}$axis. By linear fitting of the data point in Fig. $6 \mathrm{~b}$, the elastic modulus along the $c$-axis of the C-A-S-H is then estimated to be $120 \pm 30 \mathrm{GPa}$. 

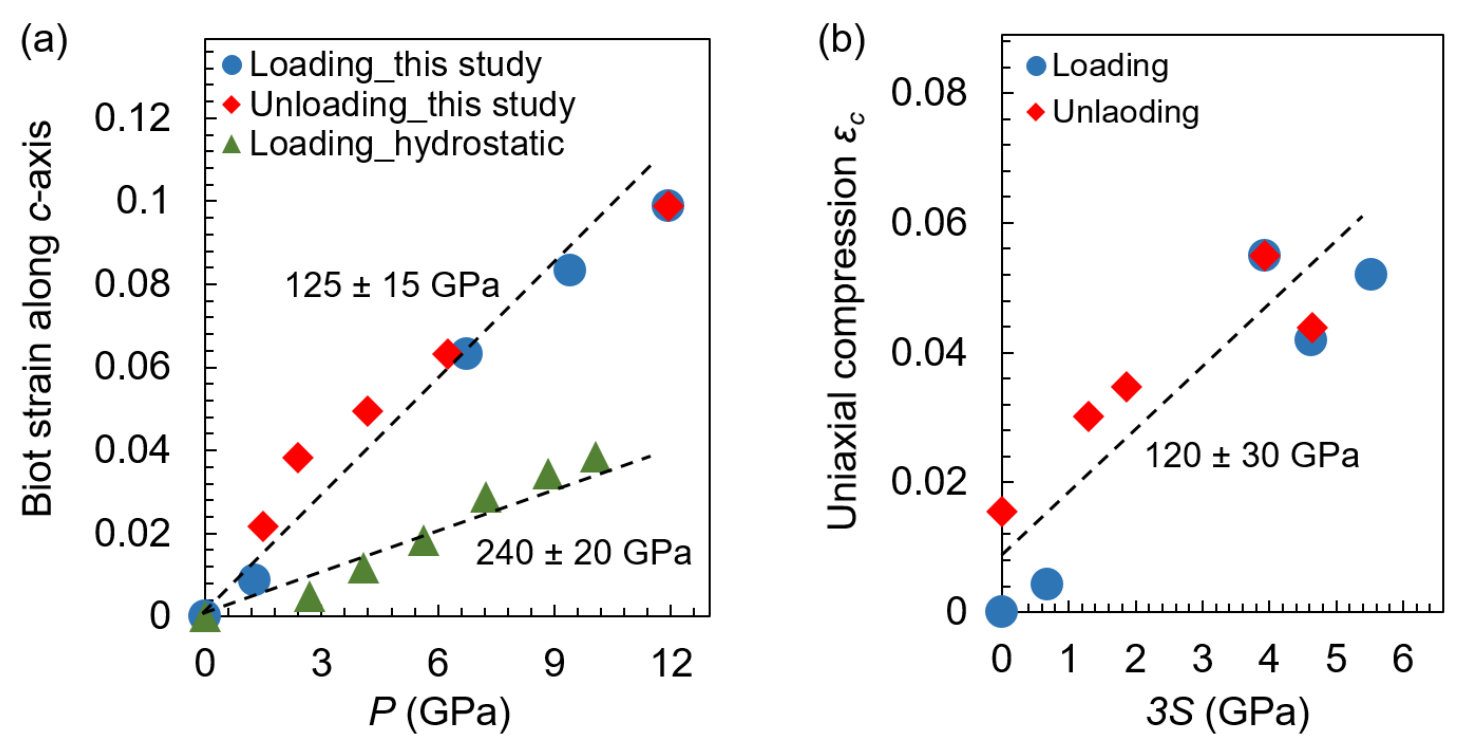

Fig. 6. (a) Biot strain along $c$-axis as a function of $P$. (b) The strain due to uniaxial compression, i.e., the difference between the c-axis strain in this study and the extrapolated values according to the study in hydrostatic condition, plotted as a function of the difference between $\sigma_{x x}$ and $P$. The slope of dashed lines, i.e., the incompressibility, is displayed in the plot.

\subsection{The influence of texture on the macroscale mechanical anisotropy}

Given the developed crystallographic texture of Al10 under compression, it is relevant to quantify its influence on the macroscale anisotropy, using the theoretical approach GMS. Here we consider three ODFs, i.e., the random orientation, the refined texture on the loading path at $P=1.3 \mathrm{GPa}$ (hereafter named Texture_1) and the refined texture on the loading path at the highest reached pressure $P=11.9 \mathrm{GPa}$ (hereafter named Texture_2). Two C-(A-)S-H models were used in the LHA calculation to obtain the elastic tensor of single crystal. The first model, hereafter named $\mathrm{CA}_{0.1} \mathrm{SH}$, is based on the molecular configuration of the studied sample Al10. It has a crosslinked double layer structure and negligible amount of vacancies at the bridging site. According to our previous study, its $c$ axis is comparable in stiffness to the $a b$-plane [20]. The second model, hereafter named 
$\mathrm{C}_{0.8} \mathrm{SH}$, is based on the configuration of a studied C-S-H sample with $\mathrm{Ca} / \mathrm{Si}=0.8, \mathrm{Al} / \mathrm{Si}=$ 0 , and a basal spacing of $14.5 \AA$. This large basal spacing results in a much softer behavior along the $c$-axis, as also verified in our previous study [18]. It is unclear whether a C-S-H sample would develop similar texture under the loading condition in the current study. Its elastic modulus is used here only to investigate the influence of the single crystal elastic anisotropy of C-(A-)S-H on its macroscale property when textured. The crystal configuration and elastic tensor of these two models are shown in Fig. 7.

Because the present goal is to study how the anisotropy of the single C-(A-)S-H phase affects the microscale anisotropy under the above-refined textures, the porosity of the grain packing is set to zero. The readers may refer to a homogenization model, e.g., Mori-Tanaka scheme, to estimate the moduli with certain porosities [63]. Based on the observed crystallite size of the C-(A-)S-H $[14,15,19,20]$, their shape in GMS model is approximated with oblate spheroids with axis ratio $l_{\mathrm{a}}: l_{\mathrm{b}}: l_{\mathrm{c}}=1: 1: 0.5$, although as shown previously, elastic properties of polycrystalline aggregate of grains with high aspect ratio ( 0.5 in this case) are very close to those of the polycrystal with spherical grains [42]. Thus, the nanocrystal shape has little influence on resulting macroscopic elastic tensor. 
(a)

$\mathrm{CA}_{0.1} \mathrm{SH}$
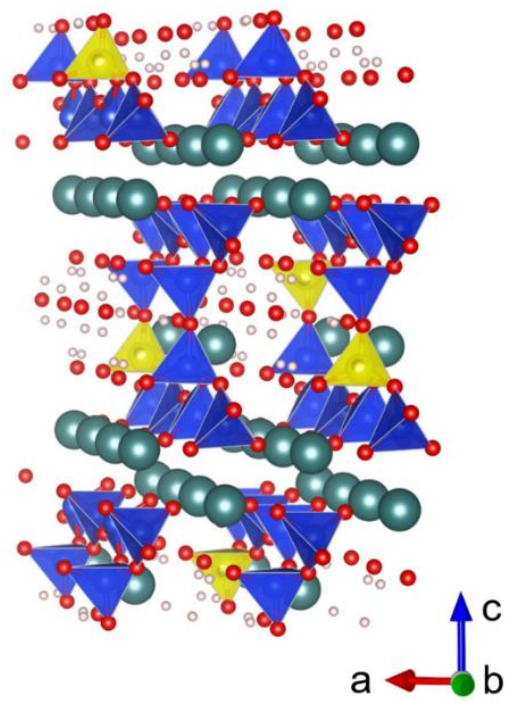

$[11$ (b)

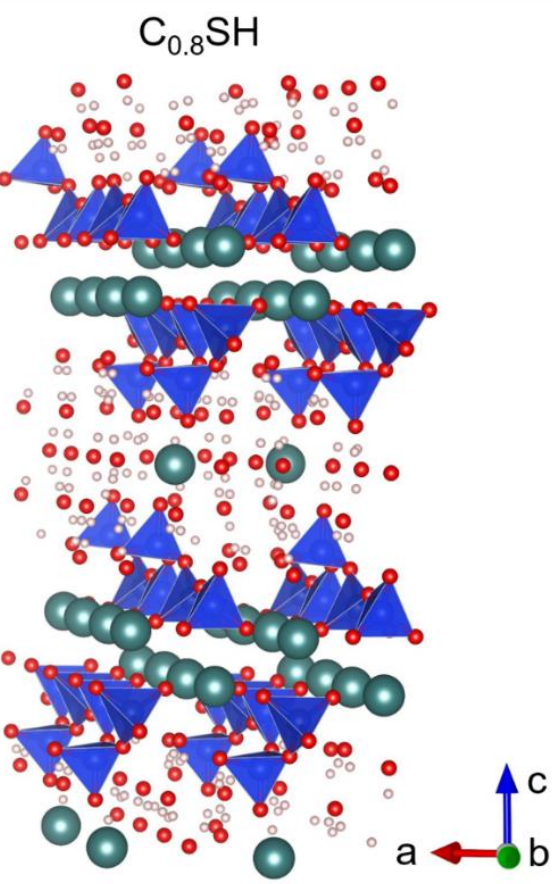

$\left[\begin{array}{cccccc}108.2 & 48.0 & 26.4 & 0.0 & 1.5 & -2.9 \\ & 108.1 & 27.1 & -1.1 & 3.2 & -6.9 \\ & & 60.5 & -0.6 & 2.4 & -2.2 \\ & & & 21.0 & -3.6 & 2.1 \\ & \multirow{2}{*}{\text { symm }} & & 19.8 & -0.7 \\ & & & & & 40.1\end{array}\right]$

Fig. 7. Molecular dynamics models of crystal structure and the corresponding stiffness tensors (in $\mathrm{GPa}$ ): (a) $\mathrm{CA}_{0.1} \mathrm{SH}$ and (b) $\mathrm{C}_{0.8} \mathrm{SH}$. The green, red and pink spheres are $\mathrm{Ca}, \mathrm{O}$ and $\mathrm{H}$, respectively. The blue and yellow tetrahedra are $\mathrm{SiO}_{4}$ and $\mathrm{AlO}_{4}$, respectively. In the notation of the stiffness tensor, the direction 2 and 3 are along $b$ - and $c$-axis, while the direction 1 is in $a b$-plane and perpendicular to $b$-axis.

From the LHA calculation, the C-A-S-H model is more isotropic, with its elastic tensor symmetry close to orthorhombic, while the C-S-H model (with larger interlayer spacing along $c$ ) is anisotropic, and its stiffness tensor symmetry is closer to hexagonal (Fig. 7). We use these stiffness tensors as input for GMS modeling, and calculate the macroscale stiffness tensor of non-textured and textured structures, as shown in Table 2. A set of characteristic macroscale elastic moduli (Young's moduli, Poisson's ratios, etc.) in 
different directions is then calculated using the macroscale stiffness tensor and given in Table 2 [64]. Relevant equations for their determination are listed in S.I.

Table 2 Macroscale elastic properties calculated using the GMS approach: components of the stiffness tensor $C_{i j}$, Young's moduli $E_{i}$, shear moduli $G_{i j}$, Poission's ratios $v_{i j}$, and bulk modulus $K$. Values are in GPa, rounded to first decimal digit. Voigt notation is used where $1,2,3,4,5$ and 6 denote $x x, y y, z z, y z, x z$ and $x y$, respectively, assuming axial symmetry.

\begin{tabular}{ccccccc}
\hline \multicolumn{5}{c}{ CA0.1SH } & \multicolumn{3}{c}{$\mathrm{C}_{0.8 \mathrm{SH}}$} \\
\hline & random & Texture_1 & Texture_2 & random & Texture_1 & Texture_2 \\
\hline$C_{11}$ & 111.0 & 112.3 & 114.2 & 84.6 & 95.6 & 103.7 \\
$C_{22}$ & 111.0 & 112.3 & 114.2 & 84.6 & 95.6 & 103.7 \\
$C_{33}$ & 111.0 & 112.0 & 114.4 & 84.6 & 68.6 & 62.8 \\
$C_{12}$ & 39.8 & 39.5 & 39.7 & 31.9 & 35.9 & 39.2 \\
$C_{13}$ & 39.8 & 39.3 & 37.3 & 31.9 & 30.4 & 28.9 \\
$C_{23}$ & 39.8 & 39.3 & 37.3 & 31.9 & 30.4 & 28.9 \\
$C_{44}{ }^{*}$ & 35.6 & 34.5 & 32.5 & 26.35 & 24.4 & 22.4 \\
$C_{55}{ }^{*}$ & 35.6 & 34.5 & 32.5 & 26.35 & 24.4 & 22.4 \\
$C_{66}{ }^{*}$ & 35.6 & 36.4 & 37.25 & 26.35 & 29.85 & 32.25 \\
$C_{r e s t}$ & 0 & 0 & 0 & 0 & 0 & 0 \\
$E_{z}$ & 90.0 & 91.7 & 96.3 & 67.1 & 54.5 & 51.1 \\
$E_{x}$ & 90.0 & 91.8 & 94.6 & 67.1 & 76.0 & 83.0 \\
$G_{x z}$ & 17.8 & 17.3 & 16.3 & 13.2 & 12.2 & 11.2 \\
$G_{x y}$ & 17.8 & 18.2 & 18.6 & 13.2 & 14.9 & 16.1 \\
$K$ & 63.5 & 63.6 & 63.5 & 49.5 & 50.4 & 51.6 \\
$v_{x y}$ & 0.26 & 0.26 & 0.27 & 0.27 & 0.27 & 0.29 \\
$v_{x z}$ & 0.26 & 0.26 & 0.24 & 0.27 & 0.32 & 0.32 \\
$v_{z x}$ & 0.26 & 0.26 & 0.24 & 0.27 & 0.23 & 0.20 \\
\hline$*$
\end{tabular}

${ }^{*}$ Two digits are introduced for $C_{44}, C_{55}$ and $C_{66}$ if necessary to comply with symmetry requirements

From Table 2, it is evident that the transversely isotropic (fiber) texture could only result in a macroscale mechanical property also possessing the transverse isotropy. When 
the single $\mathrm{C}-(\mathrm{A}-) \mathrm{S}-\mathrm{H}$ grain is nearly isotropic (i.e., $\mathrm{CA}_{0.1 \mathrm{SH}}$ ), the degree of preferred orientation has negligible influence on the macroscale property, and the material is practically isotropic for all measured ODFs. However, if the single crystal is anisotropic (i.e. $\mathrm{C}_{0.8 \mathrm{SH}}$ ), the macroscale property becomes strongly direction-dependent with the increase of pressure and texture strength. For instance, the $c$-direction of $\mathrm{C}_{0.8} \mathrm{SH}$ grains is clearly less stiff than the $a b$-plane. Thus, under Texture_1 when the $c$-direction of crystallites prefer to align along $Z$-direction, the polycrystalline aggregate has $C_{33}$ that is $\sim 25 \%$ less than $C_{11} \approx C_{22}$. Under Texture_2, when the degree of the nanocrystal alignment increases, $C_{33}$ is $\sim 35 \%$ less than $C_{11} \approx C_{22}$. Additionally, there is an increase in shear modulus $G_{x y}$, but a decrease in $G_{x z}$, compared to random crystal orientation case. It is also predicted that a definite change in the Poisson's ratio between the axial (Z-direction) and the transverse ( $X$ - and $Y$-plane) directions will occur, although the in-plane Poisson's ratio $\left(v_{x y}\right)$ will practically remain constant.

\section{Discussion and conclusions}

The results presented herein demonstrate that the randomly packed C-A-S-H nanocrystals develop a preferred orientation upon compressive loading, which was never observed in the reported hydrostatic loading study of C-(A-)S-H [18,20,65]. An exemplar comparison is shown in Fig. 8a and Fig. 8b. Under completely hydrostatic load of $P=5.6 \mathrm{GPa}$, the azimuthal distribution of the diffraction intensity on (002) and ( $h k 0)$ Debye rings is exactly the same as at the ambient condition. Whereas in this study, at comparable pressure $P=$ 6.7 GPa (but with a deviatoric stress $S=0.77 \mathrm{GPa}$ ), the (001) plane normals preferably occupy the orientation parallel to the compression direction. Such phenomenon strongly 
indicates that the deviatoric stress initiates the reorientation (Fig. 8c). This texture is conserved when the load is completely removed. This observation is consistent with a recent molecular scale study, where the inter-particle cohesion of C-S-H is maximized when the adjacent layer structures are aligned in a parallel way [66,67].

(a)

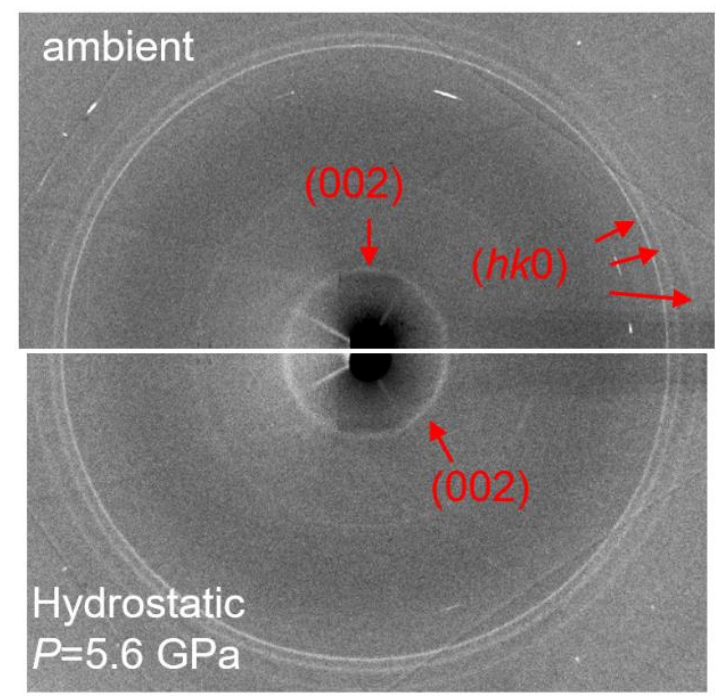

(c)
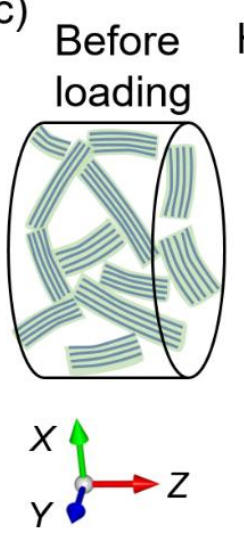

(b)

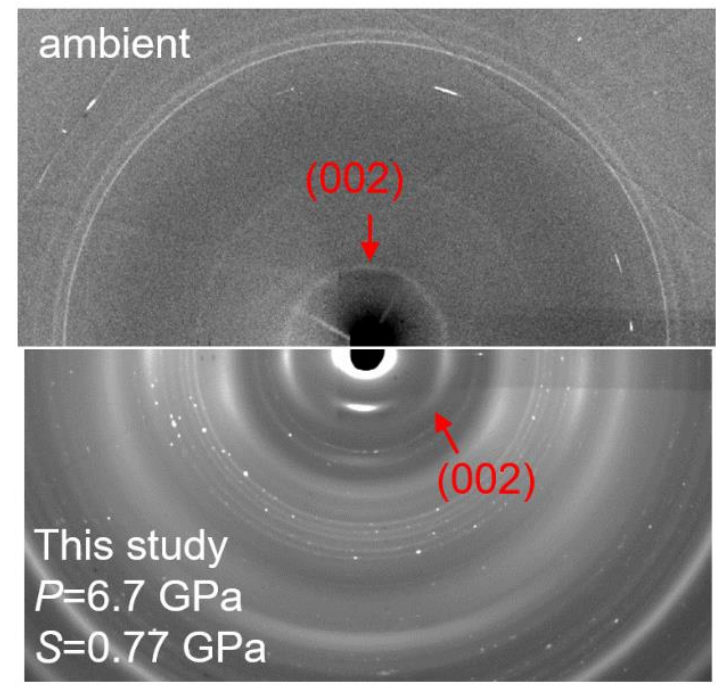

(d)

non-oriented oriented layer bulk volume

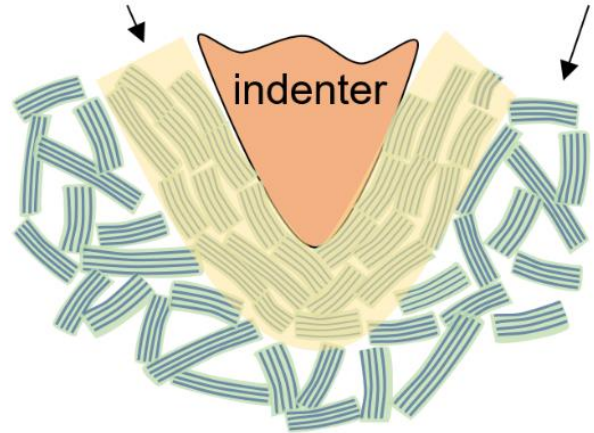

Fig. 8. Comparison of texture development under (a) hydrostatic and (b) non-hydrostatic condition, both with $P$ near $6 \mathrm{GPa}$. For viewing convenience, the diffraction images at elevated pressure are vertical flipped using half of the image with $\eta=0^{\circ}$ to $180^{\circ}$.

Schemes of (c) nanocrystals orientation in hydrostatic and uniaxial loading experiments, and (d) hypothetic orientated C-(A-)S-H layer (highlighted) near the tip of an indenter. 
The nanogranular theory of concrete creep postulates that the creep of concrete under loading is due to permanent microstructural deformation that may involve the reorientation of the C-(A-)S-H nano-morphology that results in a tighter local packing density $[2,69]$. This hypothesis lacks direct experimental support from the nano-structure study. The results presented herein provide direct evidence that the reorientation of $\mathrm{C}-(\mathrm{A}-) \mathrm{S}-\mathrm{H}$ nanocrystals is indeed possible when driven by deviatoric stress, and may progress between adjacent particles via a rotation/glide mechanism. In the concrete matrix, there exist numerous local regions with volumetric and elastic discontinuities between $\mathrm{C}-(\mathrm{A}-) \mathrm{S}-\mathrm{H}$ matrix and aggregates and/or crystals (e.g., portlandite and ettringite). A localized stress concentration in these regions may result in a rapid reorientation of C-(A-)S-H nanocrystals that significantly contributes to the so-called short-term volumetric creep [69,70]. Despite the localized rapid reorientation, a continuous shear gliding may also contribute to longterm inelastic deformation, which does not necessarily require a high deviatoric stress. Such phenomenon is predicted in a recent molecular scale study on C-S-H layer structure [68]. However, the minimum stress needed to initiate the glide, and its dependence on the inter-particle distance and surface-to-surface angle, remains yet to be determined.

Development of texture of the C-(A-)S-H nanocrystals may also be significant in the interpretation of the results obtained by the micro- and nano-mechanical measurements of cement-based material, e.g., the case of nanoindentation. The average compression exerted on C-(A-)S-H at the onset of unloading (or by definition the indentation hardness $H$ ) is at the scale of a few GPa [26,69], and the maximum shear near the indent can be even higher due to the stress concentration [71]. According to the results presented herein, such strong compression would induce preferred orientation of $\mathrm{C}-(\mathrm{A}-) \mathrm{S}-\mathrm{H}$ at the indent front, as 
illustrated in Fig. 8c. The existing literature almost exclusively presumes that the indentation measures the property of the bulk volume; however, our results raise the awareness that this oriented layer may behave differently from the bulk matrix. As shown in the previous section, when the $\mathrm{C}$-(A-) $\mathrm{S}-\mathrm{H}$ nanocrystals are least stiff along the $c$-axis, the indentation result may underestimate the stiffness of the $\mathrm{C}-(\mathrm{A}-) \mathrm{S}-\mathrm{H}$, as it measures the elasticity along the soft direction of the oriented nanocrystals. This is more likely to happen when the interlayer spacing of the studied C-(A-)S-H is larger. It should be noted that, since the re-orientation is will develop at the indent front, it is expected to influence indent hardness more significantly than the indentation modulus.

The previous HP-XRD studies measured only the bulk modulus of C-(A-)S-H. This study provides the first estimations of the elastic modulus along the $c$-axis, i.e. $120 \pm 30$ GPa for the studied C-A-S-H sample; it is in good agreement with the LHA calculation using CSH-FF force field, i.e. $C_{33}=120 \mathrm{GPa}$ in Fig. 7a. Note that the elasticity along $c$-axis depends largely on the interlayer spacing and the silicate chain crosslinking, as shown in our previous work [18,20]. For future study, the $(h k 0)$ diffractions rings should be better measured, so that the non-hydrostatic deformation along the $a b$-plane is quantified. This may require the reduction of gasket signal, and the use of a diffraction instrument with higher resolution in the range of the $(h k 0)$ rings.

The synthetic C-(A-)S-H is different from the C-(A-)S-H in actual cement paste, in terms of crystallinity and meso-scale morphology. Future work will include parallel measurements on hydrated paste samples. To summarize, this work reports the first experimental observation on the preferred orientation formation of C-A-S-H nanocrystallites, as driven by deviatoric stress. The layered $\mathrm{C}-\mathrm{A}-\mathrm{S}-\mathrm{H}$ structure prefers to 
align with layers perpendicular to the principal compression load. This texture is preserved when C-A-S-H aggregate is completely unloaded. This preferred orientation results in a transversely isotropy of macroscale elastic properties that are quantified based on observed preferred orientations and single crystal elastic properties derived from molecular dynamics models.

\section{Supporting Information}

The Supporting Information is available online as a separate file: the complete set of raw diffraction images on the loading and unloading paths; the equations used to calculate the macroscale mechanical properties.

\section{Acknowledgments}

This work is funded by the Republic of Singapore's National Research Foundation through a grant to the Berkeley Education Alliance for Research in Singapore (BEARS) for the Singapore-Berkeley Building Efficiency and Sustainability in the Tropics (SinBerBEST) Program. The Advanced Light Source is supported by the Director, Office of Science, Office of Basic Energy Sciences, of the U.S. Department of Energy under Contract No. DE-AC02-05CH11231. Sample preparation of this research was partially supported by COMPRES, the Consortium for Materials Properties Research in Earth Sciences under NSF Cooperative Agreement EAR 1606856. Martin Kunz (beamline sciencetist of ALS beamline 12.2.2) is acknowledged for helping with the high pressure experiment and the manuscript preparation. H.R. Wenk is appreciative for support from NSF (EAR 1343908). 
Guoqing Geng acknowledges additional support through the Chinese Scholarship Council (file No. 201206090127).

\section{References}

1. P.K. Mehta, P.J.M. Monteiro, Concrete Microstructure, Properties, and Materials, $4^{\text {th }}$ edition, McGraw-Hill Companies: New York City (2014).

2. H.M. Jennings, Refinements to colloid model of CSH in cement: CM-II, Cem. Concr. Res. 38 (2008) 275-289.

3. I.G. Richardson, G.W. Groves, Models for the composition and structure of calcium silicate hydrate $(\mathrm{C}-\mathrm{S}-\mathrm{H})$ gel in gardened tricalcium silicate pastes, Cem. Concr. Res. 22 (1992) 1001-1010.

4. J.J. Chen, J.J. Thomas, H.F. Taylor, H.M. Jennings, solubility and structure of calcium silicate hydrate, Cem. Concr. Res. 34 (2004) 1499-1519.

5. X. Pardal, F. Brunet, T. Charpentier, I. Pochard, A. Nonat, ${ }^{27} \mathrm{Al}$ and ${ }^{29} \mathrm{Si}$ solid-state NMR characterization of calcium-aluminosilicate-hydrate, Inorg. Chem. 51 (2012) 1827-1836.

6. E. L'Hôpital, B. Lothenbach, D. A. Kulik, K. Scrivener, Influence of calcium to silica ratio on aluminum uptake in calcium silicate hydrate., Cem. Concr. Res. 2016, 85, 111-121.

7. M.D. Jackson, J. Moon, E. Gotti, R. Taylor, S.R. Chae, M. Kunz, A.H. Emwas, C. Meral, P. Guttmann, P. Levitz, H.R. Wenk, Material and elastic properties of Altobermorite in ancient roman seawater concrete, J. Am. Ceram. Soc. 96 (2013) 2598-2606.

8. M.D. Jackson, S.R. Chae, S.R. Mulcahy, C. Meral, R. Taylor, P. Li, A.H. Emwas, J. Moon, S. Yoon, G. Vola, H.R. Wenk, Unlocking the secrets of Al-tobermorite in roman seawater concrete, Am. Mineral. 98 (2013) 1669-1687.

9. M.D. Jackson, S.R. Mulcahy, H. Chen, Y. Li, Q. Li, P. Cappelletti, H.R. Wenk. Phillipsite and Al-tobermorite mineral cements produced through low-temperature water-rock reactions in roman marine concrete, Am. Mineral. 102 (2017) 14351450.

10. S. Merlino, E. Bonaccorsi, T. Armbruster, The real structure of tobermorite 11A: normal and anomalous forms, OD character and polytypic modifications., Eur. J. Mineral. 13 (2001) 577-590.

11. E. Bonaccorsi, S. Merlino, A.R. Kampf, The crystal structure of tobermorite $14 \AA$ (plombierite), a C-S-H Phase, J. Am. Ceram. Soc. 88 (2005) 505-512. 
12. I.G. Richardson, Tobermorite/jennite-and tobermorite/calcium hydroxide-based models for the structure of $\mathrm{CSH}$ : applicability to hardened pastes of tricalcium silicate, $\beta$-dicalcium silicate, Portland cement, and blends of Portland cement with blast-furnace slag, metakaolin, or silica fume, Cem. Concr. Res. 34 (2004) 17331777.

13. S. Grangeon, F. Claret, C. Roosz, T. Sato, S. Gaboreau, Y. Linard, Structure of nanocrystalline calcium silicate hydrates: insights from X-ray diffraction, synchrotron X-ray absorption and nuclear magnetic resonance, J. Appl. Crystallogr. 49 (2016) 771-783.

14. K. Garbev, G. Beuchle, M. Bornefeld, L. Black, P Stemmermann, Cell dimensions and composition of nanocrystalline calcium silicate hydrate solid solutions. Part 1: synchrotron- based x- ray diffraction, J. Am. Ceram. Soc. 91 (2008) 3005-3014.

15. G. Renaudin, J. Russias, F. Leroux, F. Frizon, C. Cau-dit-Coumes, Structural characterization of $\mathrm{C}-\mathrm{S}-\mathrm{H}$ and $\mathrm{C}-\mathrm{A}-\mathrm{S}-\mathrm{H}$ samples - Part I: long-range order investigated by Rietveld analyses, J. Solid State Chem. 182 (2009) 3312-3319.

16. I.G. Richardson, Model structures for C-(A)-S-H (I), Acta Cryst. B. 70 (2014) 903923.

17. A. Kumar, B.J. Walder, A. Kunhi Mohamed, A. Hofstetter, B. Srinivasan, A.J. Rossini, K. Scrivener, L Emsley, P. Bowen, The atomic-level structure of cementitious calcium silicate hydrate, J. Phys. Chem. C. 121 (2017) 17188-17196.

18. G. Geng, R.J. Myers, M.J. Abdolhosseini Qomi, P.J.M. Monteiro, Densification of the interlayer spacing governs the nanomechanical properties of calcium-silicatehydrate, Sci. Rep. 7 (2017) 10986.

19. F. Battocchio, P.J.M. Monteiro, H.R. Wenk, Rietveld refinement of the structures of 1.0 CSH and 1.5 CSH, Cem. Concr. Res. 42 (2012) 1534-1548.

20. G. Geng, R.J. Myers, J. Li, R. Maboudian, C. Carraro, D.A. Shapiro, P.J.M. Monteiro, Aluminum-induced dreierketten chain cross-links increase the mechanical properties of nanocrystalline calcium aluminosilicate hydrate, Sci. Rep. 7 (2017) 44032.

21. G. Geng, R. Taylor, S. Bae, D. Hernández-Cruz, D.A. Kilcoyne, A.H. Emwas, P.J.M. Monteiro, Atomic and nano-scale characterization of a 50-year-old hydrated $\mathrm{C}_{3} \mathrm{~S}$ paste, Cem. Concr. Res. 77 (2015) 36-46.

22. T.C. Powers, T.L. Brownyard, Studies of the physical properties of hardened portland cement paste, ACI J. Proc. 43 (1946) 101-132,

23. F.H. Wittmann The structure of hardened cement paste - a basis for a better understanding of the materials properties, In Proc. Conf. on Hydraulic Cement Pastes: Their Structure and Properties, Sheffield (1976).

24. R.F. Feldman, P.J. Sereda, A model for hydrated Portland cement paste as deduced from sorption-length change and mechanical properties, Mater. Struct. 1 (1968) 509-520. 
25. L.B. Skinner, S.R. Chae, C.J. Benmore, H.R. Wenk, P.J.M. Monteiro, Nanostructure of calcium silicate hydrates in cements, Phys. Rev. Lett. 104 (2010) 195502.

26. F.J. Ulm, G. Constantinides, F.H. Heukamp, Is Concrete a poromechanics materials? - a multiscale investigation of poroelastic properties, Mater. Struct. 37 (2004) 4358.

27. E. Tajuelo Rodriguez, I.G. Richardson, L. Black, E. Boehm-Courjault, A. Nonat, J. Skibsted, Composition, silicate anion structure and morphology of calcium silicate hydrates $(\mathrm{CSH})$ synthesised by silica-lime reaction and by controlled hydration of tricalcium silicate $\left(\mathrm{C}_{3} \mathrm{~S}\right)$, Adv. Appl. Ceram. 114 (2010) 362-371.

28. M.J. Abdolhosseini Qomi, K.J. Krakowiak, M. Bauchy, K.L. Stewart, R. Shahsavari, D. Jagannathan, D.B, Brommer, A. Baronnet, M.J. Buehler, S. Yip, F.J. Ulm, Combinatorial molecular optimization of cement hydrates, Nat. Commun. (2014) 5 .

29. M.J. Abdolhosseini Qomi, F.J. Ulm, R.J.M. Pellenq, Evidence on the dual nature of aluminum in the calcium- silicate- hydrates based on atomistic simulations, $\mathrm{J}$. Am. Ceram. Soc. 95 (2012) 1128-1137

30. L. Pegado, C. Labbez, S.V. Churakov, Mechanism of aluminium incorporation into C-S-H from ab initio calculations, J Mater Chem. A 2 (2014) 3477-3483.

31. R. Shahsavari, M.J. Buehler, R.J.M. Pellenq, F.J. Ulm, First- principles study of elastic constants and interlayer interactions of complex hydrated oxides: Case study of tobermorite and jennite, J. Am. Ceram. Soc. 92 (2009) 2323-2330.

32. C.C. Dharmawardhana, A. Misra, W.Y. Ching, Quantum mechanical metric for internal cohesion in cement crystals, Sci. Rep. (2014) 4.

33. D. Hou, H. Ma, Y. Zhu, Z. Li. Calcium silicate hydrate from dry to saturated state: structure, dynamics and mechanical properties, Acta Mater. 67 (2014) 81-94.

34. H.R.. Wenk, P. Van Houtte. Texture and anisotropy, Rep. Prog. Phys. 67 (2004) 1367-1428.

35. D.P. Bentz Three-dimensional computer simulation of portland cement hydration and microstructure development, J. Am. Ceram. Soc. 80 (1997) 3-21.

36. K. Van Breugel. Numerical simulation of hydration and microstructural development in hardening cement-based materials (I) theory, Cem. Concr. Res. 25 (1995) 319-331.

37. S. Bishnoi, K.L. Scrivener. $\mu$ ic: a new platform for modelling the hydration of cements, Cem. Concr. Res. 39 (2009) 266-274.

38. K. Ioannidou, K.J. Krakowiak, M. Bauchy, C.G. Hoover, E. Masoero, S. Yip, F.J. Ulm, P. Levitz, R.J.M. Pellenq, E. Del Gado, Mesoscale texture of cement hydrates, Proc. Natl. Acad. Sci. U.S.A. 113 (2016) 2029-2034.

39. S. Hirsekorn, Elastic properties of polycrystals - a review, Text. Microstruct. 12 (1990) 1-14. 
40. H.J. Bunge, R. Kiewel, T. Reinert, L. Fritsche, Elastic properties of polycrystals influence of texture and stereology, J. Mech. Phys. Solids. 48 (2000) 29-66.

41. S. Matthies, GEO-MIX-SELF calculations of the elastic properties of a textured graphite sample at different hydrostatic pressures, J. Appl. Crystallogr. 45 (2012) $1-16$.

42. R.N. Vasin, H.R. Wenk, W. Kanitpanyacharoen, S. Matthies, R. Wirth, Elastic anisotropy modeling of kimmeridge shale, J. Geophys. Res. B: Solid Earth. 118 (2013) 3931-3956.

43. H.R. Wenk, I. Lonardelli, S. Merkel, L. Miyagi, J. Pehl, S. Speziale, C.E. Tommaseo, Deformation textures produced in diamond anvil experiments, analysed in radial diffraction geometry, J. Phys. Condens. Matter. 18 (2006) S933.

44. R. Gonzalez-Teresa, J.S. Dolado, A. Ayuela, J.C. Gimel, Nanoscale texture development of CSH gel: a computational model for nucleation and growth, Appl. Phys. Lett. 103 (2013) 234105.

45. R.J. Myers, E. L'Hôpital, J.L. Provis, B. Lothenbach, Effect of temperature and aluminium on calcium (alumino) silicate hydrate chemistry under equilibrium conditions, Cem. Concr. Res. 68 (2015) 83-93.

46. M. Kunz, A.A. MacDowell, W.A. Caldwell, D. Cambie, R.S. Celestre, E.E. Domning, R.M. Duarte, A.E. Gleason, J.M. Glossinger, N. Kelez, D.W. Plate, A beamline for high pressure studies at the advanced light source with a superconducting bending magnet as the source, J. Syn. Rad. 12 (2005) 650-658.

47. I. Kantor, V. Prakapenka, A. Kantor, P. Dera, A. Kurnosov, S. Sinogeikin, N. Dubrovinskaia, L. Dubrovinsky, BX90: a new diamond anvil cell design for X-ray diffraction and optical measurements, Rev. Sci. Instrm. (2012) 83125102.

48. S. Merkel, T. Yagi, X-ray transparent gasket for diamond anvil cell high pressure experiments, Rev. Sci. Instrm. 76 (2005) 046109.

49. A. Dewaele, P. Loubeyre, M. Mezouar, Equations of state of six metals above 94 GPa, Phys. Rev. B 70 (2004) 094112.

50. A.K. Singh, The lattice strains in a specimen (cubic system) compressed nonhydrostatically in an opposed anvil device, J. Appl. Phys. 73 (1993) 4278-4286.

51. S.M. Dorfman, S.R. Shieh, T.S. Duffy, Strength and texture of Pt compressed to 63 GPa, J. Appl. Phys. 117 (2015) 065901.

52. H.R. Wenk, L. Lutterotti, P. Kaercher, W. Kanitpanyacharoen, L. Miyagi, R.N. Vasin, Rietveld texture analysis from synchrotron diffraction images: II. Complex multiphase materials and diamond anvil cell experiments, Powder Diffr. 29 (2014) 172-192

53. H. M. Rietveld, A profile refinement method for nuclear and magnetic structures, J. Appl. Crystal. 2 (1969) 65-71. 
54. L. Lutterotti, R. Vasin, H.R. Wenk, Rietveld texture analysis from synchrotron diffraction images. I. Calibration and basic analysis, Powder Diffr. 29 (2014) 7684.

55. S. Matthies, G.W. Vinel, K. Helming, Standard distributions in texture analysis: maps for the case of cubic-orthorhombic symmetry, Akademie-Verlag Berlin. (1987) 1.

56. S. Matthies, G.W. Vinel, On the reproduction of the orientation distribution function of texturized samples from reduced pole figures using the conception of a conditional ghost correction, Phys. Status Solidi B (1982) 112.

57. H.R. Wenk, P.J.M. Monteiro, M. Kunz, K. Chen, N. Tamura, L. Lutterotti, J. Del Arroz, Preferred orientation of ettringite in concrete fractures. J. Appl. Crystal. 42 (2009) 429-432.

58. S. Matthies, H.G. Priesmeyer, M.R. Daymond, On the diffractive determination of single-crystal elastic constants using polycrystalline samples, J. Appl. Crystal. 34 (2001) 585-601.

59. P.R. Morris, elastic constants of polycrystals, Int. J. Eng. Sci. 8 (1970) 49-61.

60. J.D. Gale, GULP: A computer program for the symmetry-adapted simulation of solids, Faraday Trans. 93 (1997) 629-637.

61. R. Shahsavari, R.J.M. Pellenq, F.J. Ulm, Empirical force fields for complex hydrated calcio-silicate layered materials, PCCP. 13 (2011) 1002-1011.

62. R.T. Cygan, J.J. Liang, A.G. Kalinichev, Molecular models of hydroxide, oxyhydroxide, and clay phases and the development of a general force field, J. Phys. Chem. B 108 (2004) 1255-1266.

63. G. Constantinides, F.J. Ulm, The nanogranular nature of C-S-H, J. Mech. Phys. Solids 55 (2007) 64-90.

64. V. Lubarda, M. Chen, On the elastic moduli and compliances of transversely isotropic and orthotropic materials, J. Mech. Mater. Struct. 3 (2008) 153-171.

65. J.E. Oh, S.M. Clark, H.R. Wenk, P.J.M. Monteiro, Experimental determination of bulk modulus of $14 \AA$ tobermorite using high pressure synchrotron x-ray diffraction, Cem. Concr. Res. 42 (2012) 397-403.

66. S. Masoumi, H. Valipour, M.J. Abdolhosseini Qomi, Interparticle interactions in colloidal systems: toward a comprehensive mesoscale model, ACS Appl. Mater. Interfaces 9 (2017) 27338-27349.

67. S. Masoumi, H. Valipour, M.J. Abdolhosseini Qomi, Intermolecular forces between nanolayers of crystalline calcium-silicate-hydrates in aqueous medium, $\mathrm{J}$. Phys. Chem. C 121 (2017) 5565-5572.

68. A, Morshedifard, S. Masoumi, M.J. Abdolhosseini Qomi, Nanoscale origins of creep in calcium silicate hydrates, Nat. Commun. 9 (2018) 1785.

69. M. Vandamme, F.J. Ulm, Nanogranular origin of concrete creep, Proc. Natl. Acad. Sci. U.S.A. 106 (2009) C10552-10557. 
70. Z.P. Bažant, J.C. Chern, Double-power logarithmic law for concrete creep, Cem. Concr. Res. 14 (1984) 793-806.

71. A.C. Fischer-Cripps, Elastic indentation stress fields, Introd. Contact Mech. (2007) 77-100. 


\section{Supporting Information for}

\section{Preferred Orientation of Calcium Aluminosilicate Hydrate Induced by Confined}

\section{Compression}

Guoqing Geng ${ }^{1,2^{*}}$, Roman Nikolayevich Vasin ${ }^{3}$, Jiaqi Li ${ }^{1}$, Mohammad Javad

Abdolhosseini Qomi ${ }^{4}$, Jinyuan Yan ${ }^{5}$, Hans-Rudolf Wenk ${ }^{6}$, Paulo J. M. Monteiro ${ }^{1}$

${ }^{1}$ Department of Civil and Environmental Engineering, University of California, Berkeley, California 94720, United States

${ }^{2}$ Laboratory for Waste Management, Paul Scherrer Institut, 5232 Villigen PSI, Switzerland

${ }^{3}$ Frank Laboratory of Neutron Physics, Joint Institute for Nuclear Research, Joliot-Curie 6, 141980 Dubna, Moscow Region, Russia

${ }^{4}$ The Henry Samueli School of Engineering, University of California, Irvine, California 92697, United States

${ }^{5}$ Advanced Light Source, Lawrence Berkeley National Laboratory, Berkeley, California 94720, United States

${ }^{6}$ Department of Earth and Planetary Science, University of California, Berkeley, California 94720, United States

* Corresponding author. E-mail: guoqing.geng@psi.ch. 


\section{2D diffraction patterns at other pressure values}
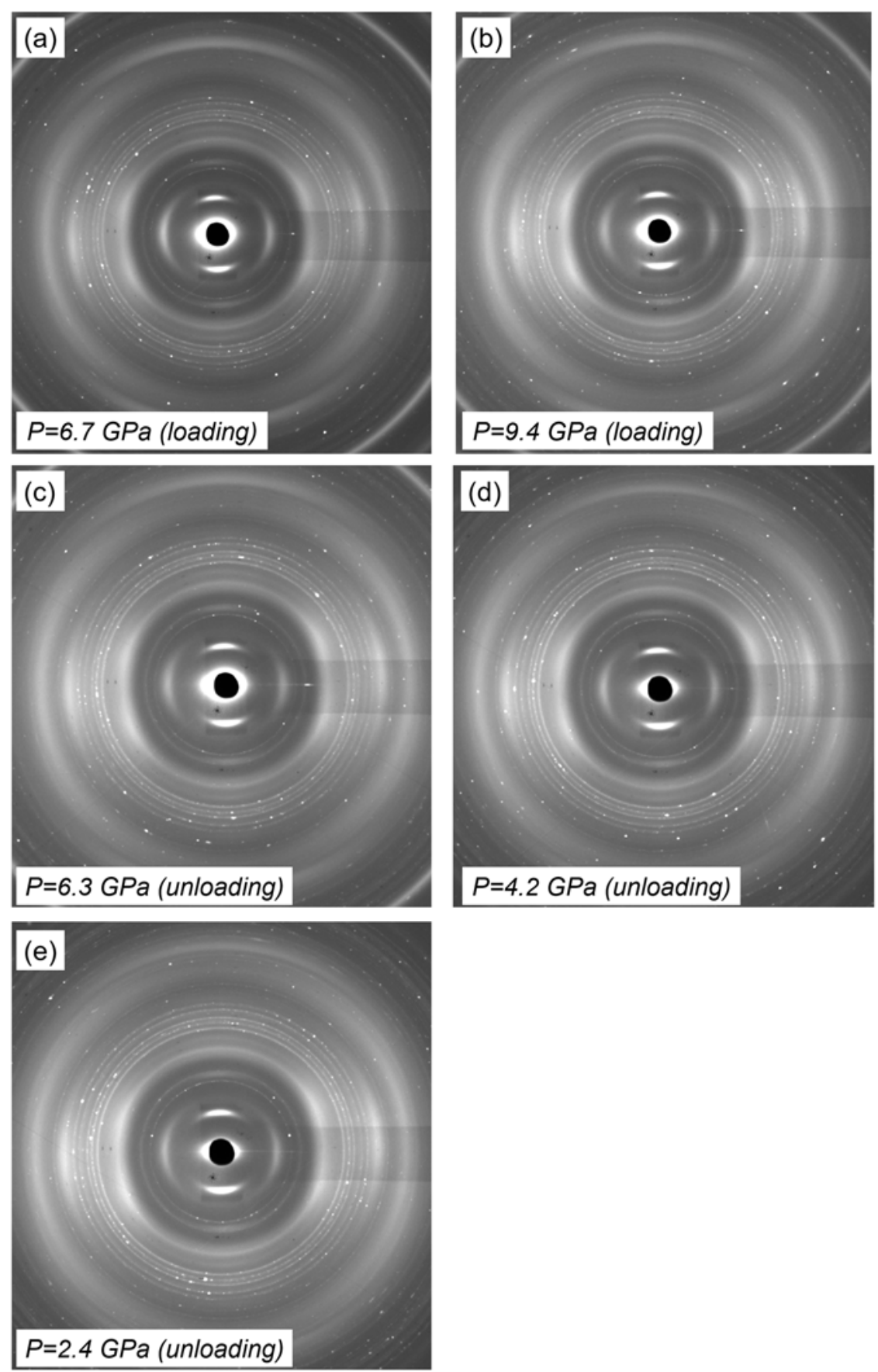

Supplementary figure 1 . The 2D diffraction patters on loading path: (a) $P=6.4 \mathrm{GPa}$, (b) $P=7.8 \mathrm{GPa}$, and on unloading path: (c) $P=5.5 \mathrm{GPa}$, (d) $P=3.6 \mathrm{GPa}$ and (e) $P$

$$
=1.6 \mathrm{GPa}
$$




\section{Equations to calculate homogenized mechanical properties}

With the elastic tensor $\boldsymbol{C}$ being calculated for the matrix, a few mechanical properties can be calculated as follows considering the transversely isotropy. The direction 1 and 2 are the transverse directions, and 3 is longitudinal.

$$
\begin{aligned}
& \text { The compliance tensor } \begin{aligned}
\boldsymbol{S} & =\boldsymbol{C}^{-1} \\
E_{3} & =1 / S_{33} \\
E_{1} & =1 / S_{22} \\
G_{13} & =0.5 / S_{44} \\
G_{12} & =0.5 / S_{66} \\
S_{22} & \left.+S_{33}+2 *\left(S_{12}+S_{13}+S_{23}\right)\right) \\
v_{12} & =-E_{1} * S_{12} \\
v_{31} & =-E_{3} * S_{13} \\
v_{13} & =-E_{1} * S_{31}
\end{aligned}
\end{aligned}
$$

